\title{
Cinética de secagem do nabo forrageiro (Raphanus sativus $\left.L_{\text {. }}\right)^{1}$
}

\author{
The drying kinetics of forage turnips (Raphanus sativus L.)
}

\author{
Kelly Aparecida de Sousa ${ }^{2 *}$, Osvaldo Resende ${ }^{3}$, Tarcísio Honório Chaves ${ }^{4}$ e Lílian Moreira Costa ${ }^{5}$
}

\begin{abstract}
Resumo - Em delineamento inteiramente casualizado com quatro repetições foi realizado um trabalho com o objetivo de determinar o modelo matemático que melhor descreve a secagem de sementes de nabo forrageiro, bem como o coeficiente de difusão e a energia de ativação em diferentes condições de ar. Sementes colhidas com teor de água de 0,36 (decimal b.s.) foram secas até 0,09 (decimal b.s.) em secador experimental com as temperaturas controladas de 30; 40; 50; 60 e $70{ }^{\circ} \mathrm{C}$ e umidades relativas de 47,$3 ; 26,2 ; 12,0 ; 10,1$ e $5,1 \%$, respectivamente. Os dados de coeficiente de difusão foram analisados por meio de análise de variância pelo teste $\mathrm{F}$ e regressão, adotando-se o nível de $5 \%$ de probabilidade. Conclui-se que dentre os modelos analisados, o que apresentou melhor ajuste para descrever as curvas de secagem do nabo forrageiro foi o de Midilli. O coeficiente de difusão efetivo aumenta com a elevação da temperatura, apresentando valores entre $3,23 \times 10^{-11}$ e $10,42 \times 10^{-11} \mathrm{~m}^{2} \mathrm{~s}^{-1}$, para faixa de temperatura de 30 a $70{ }^{\circ} \mathrm{C}$. A relação entre o coeficiente de difusão e a temperatura de secagem pode ser descrita pela equação de Arrhenius, que apresenta uma energia de ativação para a difusão líquida no processo de secagem do nabo forrageiro de $24,78 \mathrm{~kJ} \mathrm{~mol}^{-1}$.
\end{abstract}

Palavras-chave - Curvas de secagem. Brassicaceae. Semente oleaginosa. Nabo forrageiro.

\begin{abstract}
A study aimed at determining the best mathematical model to describe the drying of forage-turnip seeds, along with the diffusion coefficient and activation energy under different air-conditions, was carried out in a randomized design with four replications. Seeds were harvested, having a moisture content of 0.36 (decimal bs) and then dried to 0.09 (decimal bs) in an experimental dryer at controlled temperatures of 30;40;50;60 and $70{ }^{\circ} \mathrm{C}$ and relative humidities of $47.3 ; 26.2 ; 12.0 ; 10.1$ and $5.1 \%$, respectively. The effective coefficient of diffusion was calculated by F-test variance analysis and regression, adopting a $5 \%$ level of probability. The conclusion was reached that among the models analyzed, that of Midilli best fit the drying curves of forage-turnip seeds. The effective diffusion coefficient increases with temperature, with values of between $3.23 \times 10^{-11}$ and $10.42 \times 10^{-11} \mathrm{~m}^{2} \mathrm{~s}^{-1}$ for a temperature range of from 30 to $70{ }^{\circ} \mathrm{C}$. The relationship between the coefficient of diffusion and drying temperature can be described by the Arrhenius equation which gives an activation energy of $24.78 \mathrm{~kJ} \mathrm{~mol}^{-1}$ for liquid diffusion in the drying process of forage-turnip seeds.
\end{abstract}

Key words - Drying curves. Brassicaceae. Oilseed. Forage turnips.

\footnotetext{
*Autor para correspondência

Recebido para publicação em 18/11/2010; aprovado em 27/05/2011

Parte da dissertação de Mestrado do primeiro autor, ao Programa de Pós-Graduação em Ciências Agrárias/PPGCA, IF Goiano-campus Rio Verde, Rio Verde-GO, Brasil, Projeto financiado pelo CNPq

${ }^{2}$ Programa de Pós-Graduação em Ciências Agrárias, IF Goiano-campus Rio Verde, GO, Brasil, kellyapsousa@yahoo.com.br

${ }_{3}^{3}$ Instituto Federal Goiano-campus Rio Verde, GO, Brasil, osvresende@yahoo.com.br

${ }^{4}$ Curso de Agronomia do IF Goiano-campus Rio Verde, GO, Brasil, tarcisiohonoriochaves@gmail.com

${ }^{5}$ Pós-Graduação em Ciências Agrárias, IF Goiano-campus Rio Verde, Rio Verde-GO, Brasil, lmctpg@yahoo.com.br
} 


\section{Introdução}

Cerca de $40 \%$ de toda a energia consumida no mundo provém de combustíveis de origem fóssil. Essas fontes são limitadas e, portanto, a busca por fontes alternativas de energia é extremamente relevante. Assim, os óleos vegetais aparecem como alternativa para a substituição dos tradicionais combustíveis originados do petróleo (FERRARI; OLIVEIRA; SCABIO, 2005).

Diversas plantas vêm sendo estudadas com objetivo de fornecimento de óleo para produção de bicombustíveis dentre estas, o nabo forrageiro está surgindo como potencial matéria-prima para a obtenção de biocombustíveis.

O nabo forrageiro pertencente à família das brassicaseas é empregado para adubação verde e rotação de culturas nas regiões sul, sudeste e centro-oeste do Brasil (CRUSCIOL et al., 2005), apresenta teores de óleo na faixa de 40\% a 54\% (DOMINGOS, 2005).

Para se obter uma armazenagem segura e eficiente dos grãos do nabo forrageiro, deve-se proceder a secagem corretamente. Para que este processo seja realizado de forma rápida, segura e econômica é fundamental conhecer e monitorar os fenômenos físicos existentes durante a secagem (MARTINAZZO et al., 2007). O estudo de sistemas de secagem, seu dimensionamento, otimização e a determinação da viabilidade de sua aplicação comercial podem ser feitos por meio de simulação matemática, cujo princípio se fundamenta na secagem de sucessivas camadas delgadas do produto, utilizando-se um modelo matemático que representa satisfatoriamente a perda de água (AFONSO JÚNIOR; CORRÊA, 1999).

A secagem de produtos higroscópicos pode ser descrita por métodos teóricos, semiteóricos e empíricos. Nos métodos teóricos normalmente se consideram as condições externas sob as quais a operação ocorre como também os mecanismos internos de transferência de energia e massa e seus efeitos. Brooker, Bakker-Arkema e Hall. (1992) citam como exemplo de modelos teóricos o fluxo hidrodinâmico e as difusões: líquida, capilar, superficial, de vapor e térmica.

Os modelos semiteóricos se baseiam na Lei de Newton para resfriamento aplicada à transferência de massa, presumindo-se que as condições sejam isotérmicas e que a resistência à transferência de água se restrinja apenas à superfície do produto (BROOKER; BAKKER-ARKEMA; HALL, 1992). Entre os modelos semiteóricos, o modelo de Dois Termos (HENDERSON, 1974), o de Henderson e Pabis (1961) e o de Page (1949), têm sido amplamente utilizados (PANCHARIYA; POPOVIC; SHARMA, 2002).

De acordo com Queiroz, Pereira e Melo (1995) o método empírico consiste em formar grupos físicos adimensionais que podem ser facilmente investigados por experimentos de laboratório e baseia-senas condições externas, como temperatura, razão da mistura e velocidade do ar de secagem. Dentre esses modelos, tem-se o de Thompson, Peart e Foster (1968) e o de Wang e Singh (1978), tradicionalmente utilizados nos estudos de secagem de produtos agrícolas e de alimentos (MARTINAZZO et al., 2007).

Recentemente têm sido realizados inúmeros trabalhos com o objetivo de identificar as características de diversos produtos agrícolas durante a secagem de oleaginosas como: soja (FREGOLENTE, et al., 2003; KRZYZANOWSKI; WEST; FRANÇA NETO, 2006), mamona (GONELI, 2008), girassol (SANTOS, 2009) e pinhão manso (ULLMANN et al., 2010).

$\mathrm{Na}$ literatura, existe carência de informações a respeito das alterações que podem ocorrer nas características das sementes de nabo forrageiro ao longo da secagem, portanto, objetivou-se neste trabalho estabelecer o modelo matemático que melhor descreve a cinética de secagem de sementes de nabo forrageiro, bem como o coeficiente de difusão e a energia de ativação em diferentes condições de ar.

\section{Material e métodos}

O trabalho foi realizado no Laboratório de Pós-colheita de Produtos Vegetais, do Instituto Federal de Educação, Ciência e Tecnologia Goiano - campus Rio Verde, GO. Foram utilizadas sementes de nabo forrageiro com teor de água inicial de 0,36 (decimal b.s.). A secagem foi realizada em diferentes condições controladas de temperatura, 30; 40; 50; 60 e $70{ }^{\circ} \mathrm{C}$ e umidade relativa, 47,$3 ; 26,2 ; 12,0 ; 10,1$ e $5,1 \%$, respectivamente. As sementes foram secas em bandejas sem perfurações contendo $0,35 \mathrm{~kg}$ de produto em delineamento inteiramente casualizado, em quatro repetições.

Para a determinação das curvas de secagem e ajustes dos modelos, estabeleceu-se o teor de água final de 0,09 (decimal b.s.). Os teores de água do produto foram determinados em estufa a $105 \pm 1{ }^{\circ} \mathrm{C}$, durante 24 horas, em três repetições, até massa constante.

Para a obtenção do equilíbrio higroscópico do nabo forrageiro foram utilizadas três repetições contendo $10 \mathrm{~g}$, mantidas nas condições de secagem apontadas anteriormente e pesadas periodicamente até a massa permanecer constante. As razões de teor de água do produto foram determinadas pela expressão:

$$
R X=\frac{X^{*}-X_{e}^{*}}{X_{i}-X_{e}^{*}}
$$

em que,

RX - razão de teor de água, adimensional; 
$\mathrm{X}^{*}$ - teor de água do produto (b.s.);

$\mathrm{X}^{*}{ }_{\mathrm{i}}$ - teor de água inicial do produto (b.s.);

$\mathrm{X}_{\mathrm{e}}^{*}$ - teor de água de equilíbrio do produto (b.s.);

Aos dados experimentais da secagem do nabo forrageiro ajustaram-se modelos matemáticos utilizados por diferentes pesquisadores como Resende et al. (2009), Goneli et al. (2009), Goneli (2008), Resende et al. (2008), Corrêa et al. (2007), Andrade et al. (2006), Corrêa et al. (2006), Corrêa, Araújo e Afonso Júnior (2003) e Afonso Junior e Corrêa (1999) para representação da secagem de produtos agrícolas conforme descritos na Tabela 1.

Para o ajuste dos modelos matemáticos aos dados experimentais de secagem, realizou-se análise de regressão não linear, pelo método Gauss-Newton, por meio do programa computacional Statistica $7.0 \AA$. Adotou-se como critério para as aproximações iniciais dos coeficientes dos modelos os valores relatados na literatura para a modelagem de outros produtos agrícolas. Para determinar o grau de ajuste para cada temperatura de secagem considerou-se a significância dos coeficientes de regressão pelo teste t, adotando o nível de 5\% de significância, a magnitude do coeficiente de determinação $\left(\mathrm{R}^{2}\right)$, os valores do erro médio relativo $(\mathrm{P})$ e do erro médio estimado $(\mathrm{SE})$ e a tendência de distribuição dos resíduos (aleatória ou tendenciosa). $\mathrm{O}$ erro médio relativo e estimado, para cada um dos modelos, foram calculados conforme as seguintes expressões:

$$
\mathrm{P}=\frac{100}{\mathrm{n}} \sum \frac{|\mathrm{Y}-\hat{\mathrm{Y}}|}{\mathrm{Y}}
$$

$$
S E=\sqrt{\frac{\sum(Y-\hat{Y})^{2}}{G L R}}
$$

em que,

$\mathrm{Y}$ - valor de RU calculado experimentalmente;

$\hat{Y}$ - valor de RU estimado pelo modelo;

n - número de observações experimentais;

GLR - graus de liberdade do modelo (observações menos o número de parâmetros do modelo).

O coeficiente de difusão efetivo para as condições de secagem foi calculado ajustando-se o modelo baseado na teoria de difusão líquida aos dados observados. Essa equação é a solução analítica para a segunda lei de Fick, considerando a forma geométrica cilíndrica com aproximação de oito termos (Equação 16), desconsiderando a contração volumétrica dos grãos segundo Brooker, Bakker-Arkema e Hall (1992), de acordo com a seguinte expressão:

$$
\mathrm{RX}=\frac{\mathrm{X}^{*}-\mathrm{X}_{\mathrm{e}}^{*}}{\mathrm{X}_{\mathrm{i}}^{*}-\mathrm{X}_{\mathrm{e}}^{*}}=\sum_{\mathrm{n}=1}^{\infty} \frac{4}{\lambda_{\mathrm{n}}^{2}} \exp \left[-\frac{\lambda_{\mathrm{n}}^{2} \cdot \mathrm{D} \cdot \mathrm{t}}{\mathrm{r}}\right]
$$

em que,

$\mathrm{t}$ - tempo de secagem;

$\mathrm{D}$ - coeficiente de difusão líquida, $\mathrm{m}^{2} \mathrm{~s}^{-1}$;

$\mathrm{r}$ - raio equivalente, $\mathrm{m}$;

\begin{tabular}{|c|c|c|}
\hline Designação do modelo & Modelo & \\
\hline $\mathrm{RX}=1+\mathrm{at}+\mathrm{Bt}^{2}$ & Wang e Sing (WANG e SING, 1978) & (2) \\
\hline$R X=a \cdot \exp (-k \cdot t)+(1-a) \exp \left(-k_{1} \cdot t\right)$ & Verma( VERMA et AL., 1985) & (3) \\
\hline$R X=\exp \left[\left[-a-\left(a^{2}+4 \cdot b \cdot t\right)^{0,5}\right] / 2 \cdot b\right]$ & Thompson (THOMPSON et al., 1968) & (4) \\
\hline $\mathrm{RX}=\exp \left(-\mathrm{k} \cdot \mathrm{t}^{\mathrm{n}}\right)$ & Page (PAGE, 1949) & $(5)$ \\
\hline $\mathrm{RX}=\exp (-\mathrm{K} . \mathrm{t})$ & Newton (LEWIS, 1921) & (6) \\
\hline $\mathrm{RX}=\mathrm{a} \cdot \exp \left(-\mathrm{k} \cdot \mathrm{t}^{\mathrm{n}}\right)+\mathrm{b} \cdot \mathrm{t}$ & Midilli (MIDILLI, 2002) & (7) \\
\hline$R X=a \cdot \exp (-k \cdot t)+c$ & Logarítmico (YAGCIOGLU et al., 1999) & (8) \\
\hline $\mathrm{RX}=\mathrm{a} \cdot \exp (-\mathrm{k} . \mathrm{t})$ & Henderson e Pabis (HENDERSON E PABIS, 1961) & (9) \\
\hline$R X=a \cdot \exp (-k \cdot t)+b \cdot \exp \left(-k_{0} \cdot t\right)+c \cdot \exp \left(-k_{1} \cdot t\right)$ & Henderson e Pabis modificado (KARATHANOS,1999) & $(10)$ \\
\hline$R X=a \cdot \exp (-k \cdot t)+(1-a) \exp (-k \cdot a \cdot t)$ & Exponencial de Dois Termos (SHARAF-ELDEE et al., 1980) & $(11)$ \\
\hline$R X=a \cdot \exp \left(-k_{0} \cdot t\right)+b \cdot \exp \left(-k_{1} \cdot t\right)$ & Dois Termos (HENDERSON, 1974) & $(12)$ \\
\hline$R X=a \cdot \exp (-k \cdot t)+(1-a) \cdot \exp (-k \cdot b \cdot t)$ & Aproximação da Difusão (KASSEM, 1998) & (13) \\
\hline
\end{tabular}

n - número de termos.

Tabela 1 - Modelos matemáticos utilizados para predizer a secagem de produtos agrícolas

em que, t: tempo de secagem, h; k, $\mathrm{k}_{\mathrm{o}}, \mathrm{k}_{1}$ : constantes de secagem, $\mathrm{h}^{-1} ; \mathrm{a}, \mathrm{b}, \mathrm{c}, \mathrm{n}$ : coeficientes dos modelos 
O raio equivalente é definido como o raio de uma esfera com volume equivalente ao do grão. O volume $(\mathrm{V})$ de cada grão foi obtido por meio da medição dos três eixos ortogonais (comprimento, largura e espessura), em trinta grãos, antes da secagem, com auxílio de um paquímetro digital, de acordo com a expressão proposta por Mohsenin (1986):

$$
\mathrm{Vg}=\frac{\pi \cdot(\mathrm{a} \cdot \mathrm{b} \cdot \mathrm{c})}{6}
$$

em que,

a - maior eixo da semente;

b - eixo médio da semente;

c - menor eixo da semente.

O coeficiente de difusão, geralmente, aumenta com a elevação da temperatura do ar de secagem (RAMESH, 2003) e esta relação pode ser descrita por meio da equação de Arrhenius:

$$
\mathrm{D}=\mathrm{D}_{\mathrm{o}} \cdot \exp \left(\frac{-\mathrm{E}_{\mathrm{a}}}{\mathrm{R} \cdot \mathrm{T}_{\mathrm{a}}}\right)
$$

em que,

$\mathrm{D}_{\mathrm{o}}$ - fator pré-exponencial;

$\mathrm{E}_{\mathrm{a}}$ - energia de ativação, $\mathrm{kJ} \cdot \mathrm{mol}^{-1}$;

$\mathrm{R}$ - constante universal dos gases, $8,134 \mathrm{~kJ} \cdot \mathrm{kmol}^{-1} \cdot \mathrm{K}^{-1}$;

$\mathrm{T}_{\mathrm{a}}$ - temperatura absoluta, $\mathrm{K}$.

Os coeficientes da expressão de Arrhenius foram linearizados com a aplicação do logaritmo na seguinte forma:

$$
\operatorname{LnD}=\operatorname{LnD}_{\mathrm{o}}-\frac{\mathrm{E}_{\mathrm{a}}}{\mathrm{RT}} \cdot \frac{1}{\mathrm{~T}_{\mathrm{a}}}
$$

Os dados foram analisados por meio de análise de variância pelo teste $\mathrm{F}$ e regressão, adotando-se o nível de $5 \%$ de significância.

\section{Resultado e discussão}

Na Figura 1 estão representadas as curvas de secagem nas diversas condições de ar para o nabo forrageiro. Verificou-se que para as temperaturas de 30; 40; 50; 60 e $70{ }^{\circ} \mathrm{C}$ os tempos de secagem das sementes foram de 19,$0 ; 9,5 ; 5,2 ; 3,0$ e 2,5 horas, respectivamente, considerando a redução do teor de água de 0,36 para 0,09 (decimal b.s.).

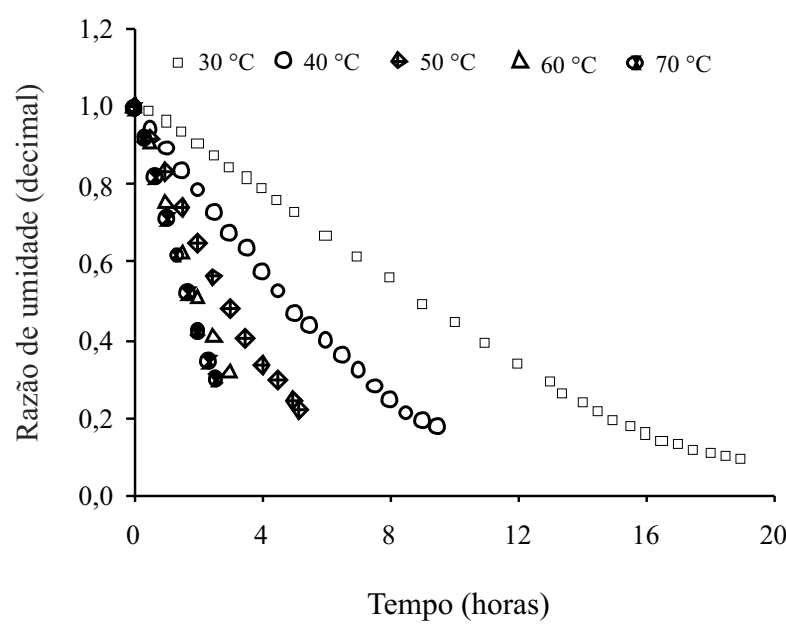

Figura 1 - Curvas de secagem para o nabo forrageiro nas temperaturas de $30 ; 40 ; 50 ; 60$ e $70{ }^{\circ} \mathrm{C}$

Pode-se observar a influência da temperatura sobre as curvas de secagem das sementes de nabo forrageiro. $\mathrm{O}$ aumento da temperatura do ar de secagem faz com que haja uma maior taxa de remoção de água da semente devido a um maior gradiente de umidade entre a semente e o ar, decrescendo o tempo necessário para reduzir o teor de água até o valor desejado, fato observado por diversos pesquisadores em produtos oleaginosos como: uva (YALDIZ; ERTEKIN; UZUN, 2001), mamona (GONELI, 2008) e pinhão manso (ULMANN et al., 2010), amiláceos como: arroz em casca (BASUNIA; ABE, 2001) e aleuroamiláceo como feijão vermelho (RESENDE et al., 2008) e feijão adzuki (ALMEIDA et al., 2009).

$\mathrm{Na}$ Tabela 2 estão representados os valores dos parâmetros estatísticos analisados para os doze modelos ajustados, durante a secagem do nabo forrageiro nas diversas condições de ar.

Em relação aos coeficientes de determinação $\left(\mathrm{R}^{2}\right)$ observa-se que somente os modelos Wang e Sing (2), Page (5), Midilli (7), Logarítmico (8) e Aproximação da Difusão (13) apresentaram valores superiores a $98 \%$ para todas as temperaturas de secagem, que de acordo com Madamba, Driscoli e Buckle (1996), indica uma representação satisfatória do fenômeno em estudo. De acordo com este pesquisador, a utilização do coeficiente de determinação como o único critério de avaliação para a seleção dos modelos não lineares não constitui um bom parâmetro para representação do fenômeno de secagem. Então, realizou-se uma análise conjunta de outros parâmetros estatísticos.

Analisando os valores obtidos referentes ao erro médio estimado (SE), nota-se que os modelos Page (5), Newton (6), Midilli (7) e Logaritmo (8) apresentaram 
Tabela 2 - Coeficientes de determinação $\left(\mathrm{R}^{2}\right)$, erros médio relativo $(\mathrm{P})$ e estimado $(\mathrm{SE})$ para os doze modelos analisados, durante a secagem do nabo forrageiro nas diversas condições de temperatura $\left({ }^{\circ} \mathrm{C}\right)$

\begin{tabular}{|c|c|c|c|c|c|c|c|c|c|c|c|c|c|c|c|}
\hline \multirow{3}{*}{$\begin{array}{l}\frac{0}{0} \\
\frac{0}{2}\end{array}$} & \multicolumn{15}{|c|}{ Temperatura $\left({ }^{\circ} \mathrm{C}\right)$} \\
\hline & \multicolumn{3}{|c|}{-----------30------------ } & \multicolumn{3}{|c|}{-----------40------------- } & \multicolumn{3}{|c|}{ - } & \multicolumn{3}{|c|}{----------60----------- } & \multicolumn{3}{|c|}{-------70--------- } \\
\hline & $\mathrm{R}^{2}$ & $\mathrm{P}$ & SE & $\mathrm{R}^{2}$ & $\mathrm{P}$ & $\mathrm{SE}$ & $\mathrm{R}^{2}$ & $\mathrm{P}$ & SE & $\mathrm{R}^{2}$ & $\mathrm{P}$ & SE & $\mathrm{R}^{2}$ & $\mathrm{P}$ & SE \\
\hline 2 & 0,99 & 6,90 & 0,002 & 0,99 & 1,60 & 0,0004 & 0,99 & 2,20 & 0,001 & 0,99 & 1,47 & 0,001 & 0,99 & 1,47 & 0,0003 \\
\hline 3 & 0,95 & 25,90 & 0,029 & 0,72 & 38,56 & 0,092 & 0,01 & 69,41 & 0,280 & 0,99 & 1,52 & 0,001 & 0,97 & 6,71 & 0,006 \\
\hline 4 & 0,95 & 25,26 & 0,028 & 0,98 & 8,70 & 0,006 & 0,98 & 7,83 & 0,005 & 0,98 & 5,11 & 0,004 & 0,97 & 6,70 & 0,005 \\
\hline 5 & 0,99 & 4,50 & 0,0012 & 0,99 & 1,98 & 0,0004 & 0,99 & 0,91 & 0,0001 & 0,99 & 0,71 & 0,0001 & 0,99 & 0,68 & 0,0001 \\
\hline 6 & 0,95 & 25,25 & 0,028 & 0,98 & 8,70 & 0,006 & 0,98 & 7,83 & 0,005 & 0,98 & 5,11 & 0,003 & 0,97 & 6,71 & 0,005 \\
\hline 7 & 0,99 & 3,04 & 0,0003 & 0,99 & 1,43 & 0,0001 & 0,99 & 0,91 & 0,0001 & 0,99 & 0,79 & 0,00009 & 0,99 & 0,46 & 0,00002 \\
\hline 8 & 0,99 & 5,61 & 0,001 & 0,99 & 1,61 & 0,0003 & 0,99 & 2,18 & 0,0005 & 0,99 & 1,38 & 0,0004 & 0,99 & 1,32 & 0,0003 \\
\hline 9 & 0,95 & 19,14 & 0,001 & 0,98 & 8,70 & 0,006 & 0,98 & 7,83 & 0,005 & 0,99 & 0,81 & 0,0001 & 0,97 & 6,71 & 0,005 \\
\hline 10 & 0,99 & 5,46 & 0,001 & 0,99 & 6,73 & 0,0043 & 0,89 & 19,36 & 0,034 & 0,96 & 5,89 & 0,012 & 0,98 & 5,28 & 0,005 \\
\hline 11 & 0,95 & 25,25 & 0,028 & 0,98 & 8,70 & 0,006 & 0,98 & 7,83 & 0,005 & 0,99 & 0,81 & 0,0001 & 0,97 & 6,71 & 0,005 \\
\hline 12 & 0,97 & 19,09 & 0,017 & 0,99 & 6,68 & 0,004 & 0,99 & 5,84 & 0,003 & 0,99 & 4,35 & 0,003 & 0,98 & 5,47 & 0,004 \\
\hline 13 & 0,99 & 7,25 & 0,002 & 0,99 & 1,87 & 0,0003 & 0,99 & 2,47 & 0,001 & 0,99 & 1,52 & 0,001 & 0,98 & 6,71 & 0,006 \\
\hline
\end{tabular}

menores valores quando comparados aos demais. Ainda na Tabela 2, analisando o erro médio relativo, observa-se que para os modelos Wang e Sing (2), Page (5), Midilli (7), Logaritmo (8), Henderson e Pabis Modificado (10) e Aproximação da Difusão (13), os valores foram inferiores a $10 \%$ para todas as temperaturas analisadas, que de acordo com Mohapatra e Rao (2005), indica uma representação adequada do fenômeno de secagem.
Então, de acordo com esses parâmetros estatísticos, os modelos Wang e Sing (2), Page (5), Midilli (7), Logarítmico (8) e Aproximação da Difusão (13) obtiveram os melhores ajustes aos dados experimentais da secagem do nabo forrageiro. $\mathrm{Na}$ Tabela 3 estão apresentadas as tendências de distribuição de resíduos para os modelos ajustados aos dados experimentais da secagem do nabo forrageiro.

Tabela 3 - Tendências de distribuição de resíduos para os modelos ajustados aos dados experimentais da secagem do nabo forrageiro para as temperaturas de $30 ; 40 ; 50 ; 60$ e $70{ }^{\circ} \mathrm{C}$

\begin{tabular}{cccccc}
\hline \multirow{2}{*}{ Modelo } & \multicolumn{5}{c}{ Temperatura $\left({ }^{\circ} \mathrm{C}\right)$} \\
\cline { 2 - 6 } & 30 & 40 & 50 & 60 & 70 \\
\hline 2 & Tendencioso & Aleatório & Tendencioso & Tendencioso & Tendencioso \\
3 & Tendencioso & Tendencioso & Tendencioso & Tendencioso & Tendencioso \\
4 & Tendencioso & Tendencioso & Tendencioso & Tendencioso & Tendencioso \\
5 & Tendencioso & Tendencioso & Aleatório & Aleatório & Aleatório \\
6 & Tendencioso & Tendencioso & Tendencioso & Tendencioso & Tendencioso \\
7 & Aleatório & Aleatório & Aleatório & Aleatório & Aleatório \\
8 & Tendencioso & Aleatório & Tendencioso & Tendencioso & Tendencioso \\
9 & Tendencioso & Tendencioso & Tendencioso & Tendencioso & Tendencioso \\
10 & Tendencioso & Tendencioso & Tendencioso & Tendencioso & Tendencioso \\
11 & Tendencioso & Tendencioso & Tendencioso & Aleatório & Tendencioso \\
12 & Tendencioso & Tendencioso & Tendencioso & Tendencioso & Tendencioso \\
13 & Tendencioso & Aleatório & Tendencioso & Tendencioso & Tendencioso \\
\hline
\end{tabular}


Verifica-se na Tabela 3 que o modelo de Midilli (7) apresentou para todas as condições de secagem uma distribuição aleatória dos resíduos, resultando assim em melhor ajuste aos dados experimentais de secagem do nabo forrageiro.

$\mathrm{Na}$ Figura 2 estão ilustradas as tendências de distribuição dos resíduos aleatórias para o modelo de Midilli $30{ }^{\circ} \mathrm{C}$ e tendenciosa para o modelo de Thompson $30{ }^{\circ} \mathrm{C}$. Os resíduos foram obtidos por meio da diferença entre os valores experimentais e os valores estimados pelo modelo.

Um modelo é considerado aleatório se os valores residuais se encontrarem próximos à faixa horizontal em torno de zero e também não formarem figuras definidas, não
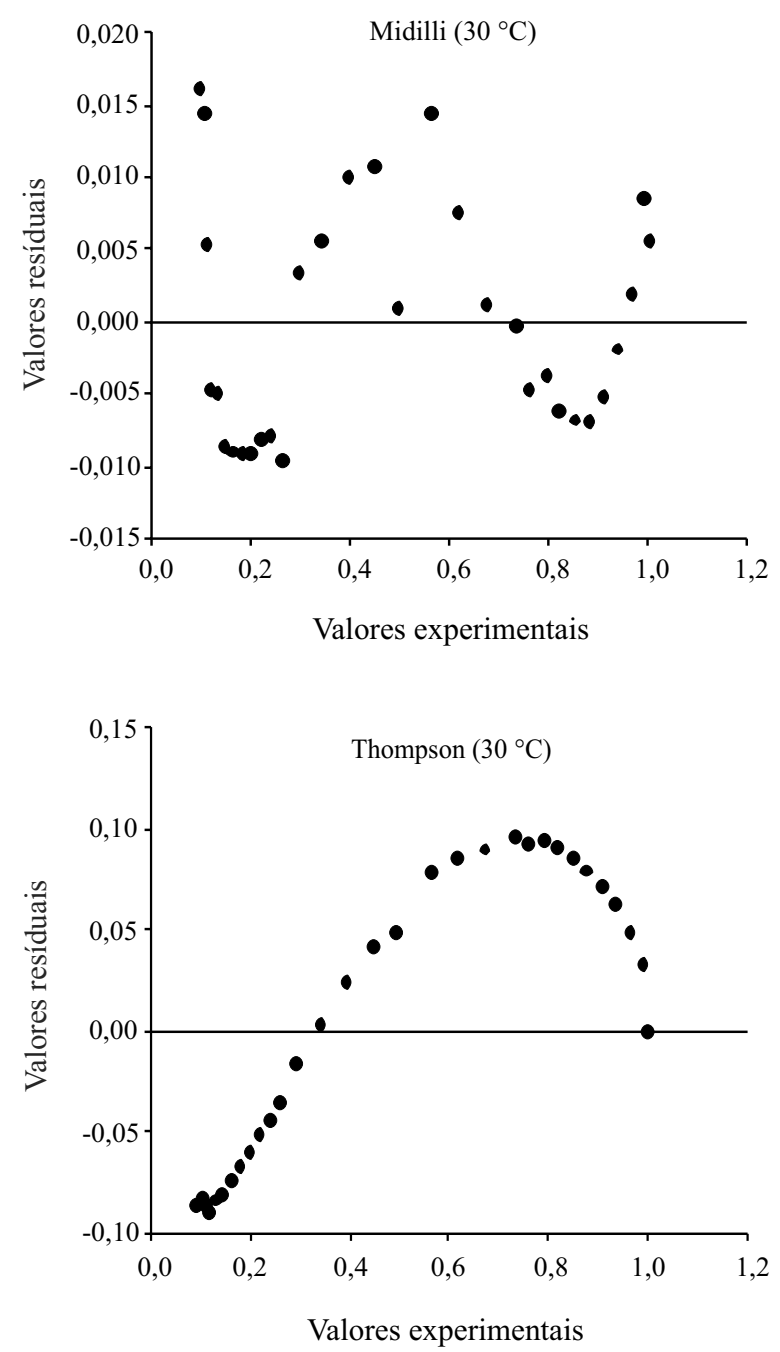

Figura 2- Tendência de distribuição dos resíduos para o modelo de Midilli $30{ }^{\circ} \mathrm{C}$ com distribuição aleatória e distribuição tendenciosa para o modelo de Thompson $30{ }^{\circ} \mathrm{C}$, durante a secagem do nabo forrageiro. indicando tendenciosidade dos resultados. Se apresentar distribuição tendenciosa, o modelo é considerado como inadequado para representar o fenômeno em questão (GONELI, 2008).

Martinazzo et al. (2007) ajustaram vários modelos aos dados experimentais da secagem de folhas de capimlimão nas temperaturas de $\left(30 ; 40 ; 50\right.$ e $\left.60^{\circ} \mathrm{C}\right)$ e a equação de Midilli dentre os modelos avaliados, foi a que se ajustou melhor aos seus dados de secagem deste produto.

Assim, para a representação gráfica das curvas de secagem (FIG. 3), utilizou-se o modelo de Midilli (7) que foi o que melhor se ajustou aos dados experimentais para representação do fenômeno de secagem. Também pode ser observado a correspondência entre os valores experimentais e estimados e o ajuste satisfatório do modelo de Midilli para descrição da secagem dos grãos de nabo forrageiro.

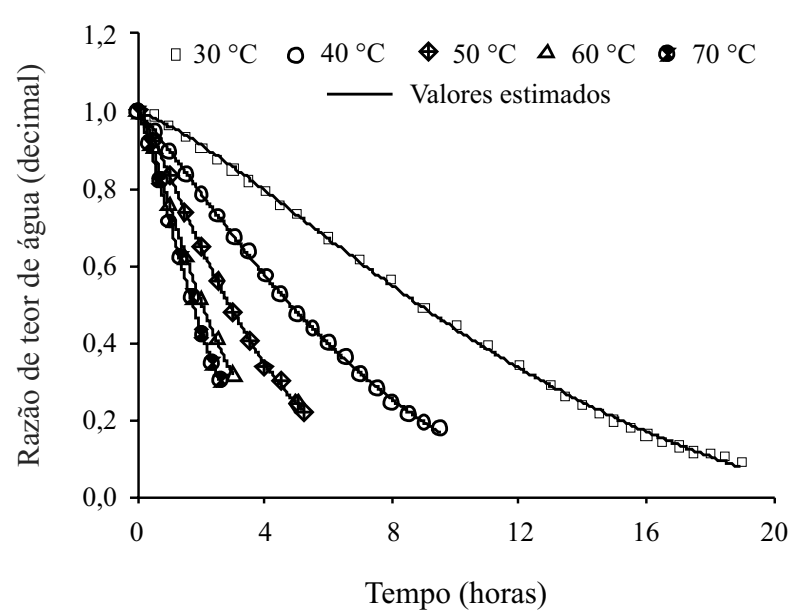

Figura 3 - Valores experimentais da razão de teor de água (RX, decimal) e estimados pelo modelo de Midilli para a secagem do nabo forrageiro nas temperaturas de $30 ; 40 ; 50 ; 60$ e $70{ }^{\circ} \mathrm{C}$

$\mathrm{Na}$ Tabela 4 são apresentados os coeficientes do modelo de Midilli ajustados aos dados experimentais da cinética de secagem do nabo forrageiro nas diferentes temperaturas.

Observou-se que a magnitude da constante de secagem (k) para o modelo de Midilli, que representa o efeito das condições externas de secagem, aumenta linearmente com a elevação da temperatura do ar de secagem (TAB. 4). Segundo Madamba, Driscoli e Buckle (1996) e Babalis e Belessiotis (2004), a constante de 
Tabela 4 - Coeficientes do modelo de Midilli ajustados para a secagem do nabo forrageiro em diferentes condições de temperatura e umidade relativa do ar com as respectivas equações

\begin{tabular}{|c|c|c|c|c|c|c|}
\hline \multirow{2}{*}{ Coeficientes } & \multicolumn{5}{|c|}{ Temperatura $\left({ }^{\circ} \mathrm{C}\right)$} & \multirow{2}{*}{ Valores médios } \\
\hline & 30 & 40 & 50 & 60 & 70 & \\
\hline $\mathrm{a}$ & $0,995^{*}$ & $0,999 *$ & $0,997 *$ & $1,002^{*}$ & $0,999 *$ & 0,998 \\
\hline $\mathrm{k}$ & $0,029^{*}$ & $0,100 *$ & $0,177^{*}$ & $0,285^{*}$ & $0,2949 *$ & $\mathrm{k}=0,007 * * \mathrm{~T}-0,180 * *$ \\
\hline $\mathrm{n}$ & $1,394 *$ & $1,173^{*}$ & $1,281^{*}$ & $1,304^{*}$ & $1,288^{*}$ & 1,289 \\
\hline $\mathrm{b}$ & $-0,005^{*}$ & $-0,008^{*}$ & $-0,001^{\mathrm{NS}}$ & $0,0044^{\mathrm{NS}}$ & $-0,028^{\mathrm{NS}}$ & $-0,008$ \\
\hline
\end{tabular}

*Significativo a $1 \%$ pelo teste $\mathrm{t}$; ${ }^{*}$ Significativo a $5 \%$ pelo teste $\mathrm{t}$; ${ }^{\text {NS }}$ Não significativo

secagem (k) pode ser utilizada como uma aproximação para caracterizar o efeito da temperatura e está relacionada à difusividade efetiva no processo de secagem no período decrescente, sendo a difusão líquida que controla o processo de secagem. Os demais coeficientes do modelo de Midilli ( $\mathrm{a}, \mathrm{n}$ e b) não apresentaram uma tendência clara em função da temperatura de secagem. Desta forma, a cinética de secagem do nabo forrageiro pode ser estimada, para a faixa de temperatura estudada, utilizando-se a seguinte expressão:

$\mathrm{RX}=0,998 \cdot \exp \left(-(0,007 \mathrm{~T}-0,180) \cdot \mathrm{t}^{1,289}\right)+(-0,008) \cdot \mathrm{t}$

em que,

$\mathrm{T}$ - temperatura de secagem $\left({ }^{\circ} \mathrm{C}\right)$;

$\mathrm{t}$ - tempo de secagem (h).

Encontram-se na Figura 4, os dados de razão de teor de água (RX), experimentais e estimados empregando-se a equação de Midilli com os valores obtidos por meio da Equação 20 e dos valores apresentados na Tabela 4. Constata-se na Figura 4 o bom ajuste deste modelo na descrição da secagem do nabo forrageiro. Entretanto, com a redução dos valores da razão de teor de água, ocorre uma maior discrepância entre os valores experimentais e estimados pelo modelo.

Na Tabela 5 estão apresentados os valores médios dos coeficientes de difusão efetivo determinados para a secagem do nabo forrageiro nas temperaturas de 30 ; $40 ; 50 ; 60$ e $70{ }^{\circ} \mathrm{C}$.

Na Figura 5 estão apresentados os valores médios dos coeficientes de difusão efetivo determinados para a secagem do nabo forrageiro na faixa de temperatura de 30 a $70{ }^{\circ} \mathrm{C}$.

Verifica-se que durante a secagem, os coeficientes de difusão apresentaram magnitudes entre $3,23 \times 10^{-11} \mathrm{e}$ $10,43 \times 10^{-11} \mathrm{~m}^{2} \mathrm{~s}^{-1}$, para a faixa de temperatura de $30 \mathrm{a}$

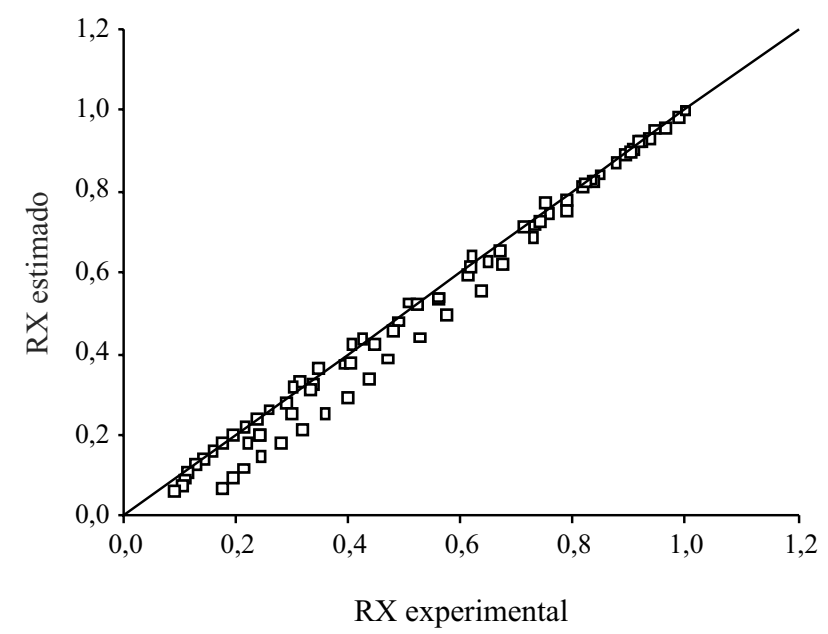

Figura 4 - Valores experimentais e estimados de razão de teor de água pela estimativa dos parâmetros da equação de Midilli para a secagem do nabo forrageiro

Tabela 5 - Resumo da análise de variância para o coeficiente de difusão efetivo das sementes de nabo forrageiro obtido para diversas temperaturas de secagem

\begin{tabular}{ccc}
\hline $\begin{array}{c}\text { Variáveis } \\
\text { analisadas }\end{array}$ & Quadrado médio & $\begin{array}{c}\text { Coeficiente de } \\
\text { variação }(\%)\end{array}$ \\
\hline $\begin{array}{c}\text { Coeficiente de } \\
\text { difusão efetivo }\end{array}$ & $33,3837 * *$ & 21,60 \\
\hline
\end{tabular}

**Significativo a $1 \%$ pelo teste $\mathrm{F}$

$70{ }^{\circ} \mathrm{C}$. Resende et al. (2005) obtiveram para o arroz em casca valores semelhantes da ordem de 5,354 x $10^{-11}$ a $24,321 \times 10^{-11} \mathrm{~m}^{2} \mathrm{~s}^{-1}$ para as temperaturas de 25 e $55^{\circ} \mathrm{C}$, respectivamente, sendo que as menores temperaturas do ar de secagem ofereceram maiores resistências internas ao transporte de água do produto. Com a elevação da 


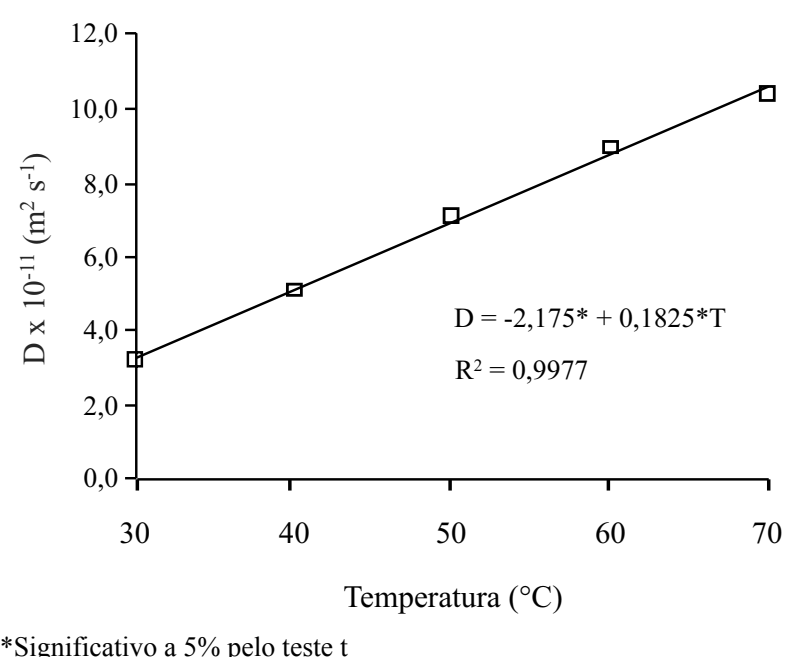

Figura 5 - Valores médios do coeficiente de difusão efetivo $\left(\mathrm{m}^{2} \mathrm{~s}^{-1}\right)$ obtidos para a secagem do nabo forrageiro nas temperaturas de $30 ; 40 ; 50 ; 60$ e $70{ }^{\circ} \mathrm{C}$

temperatura do ar de secagem, os valores do coeficiente de difusão aumentaram, indicando uma maior intensidade do fenômeno de transporte de água do interior para a periferia do grão, corroborando com Sharma e Prasad (2004), Babalis e Belessiotis (2004), Goneli et al. (2007) e Almeida et al. (2009).

O coeficiente de difusão aumentou linearmente com a elevação da temperatura do ar de secagem, e a sua influência pode ser descrita por meio da representação de Arrhenius, conforme ilustrado na Figura 6.

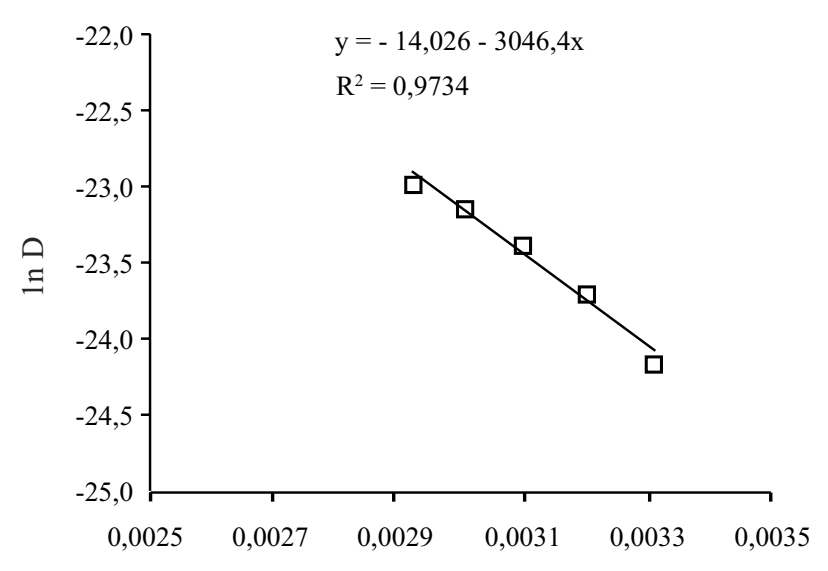

$1 / \mathrm{T}\left(\mathrm{K}^{-1}\right)$

Figura 6 - Representação de Arrhenius para o coeficiente de difusão efetivo para a secagem do nabo forrageiro nas temperaturas de $30 ; 40 ; 50 ; 60$ e $70{ }^{\circ} \mathrm{C}$
Termodinamicamente a energia de ativação é definida como a facilidade com que as moléculas de água superam a barreira de energia durante e migração do interior do produto (CORRÊA et al., 2005). A Equação 21 apresenta os coeficientes da equação ajustada para o coeficiente de difusão efetivo do nabo forrageiro, calculados de acordo com a expressão 19.

$$
\mathrm{D}=3,45 \cdot 10^{-5} \exp \left(\frac{-24776,16}{\mathrm{R} \cdot \mathrm{T}_{\mathrm{a}}}\right)
$$

Conforme verificado na Equação 21, a energia de ativação para a difusão líquida do nabo forrageiro foi de $24,78 \mathrm{~kJ} \mathrm{~mol}^{-1}$. Nos processos de secagem, quanto menor a energia de ativação maior será a difusividade de água no produto. A energia de ativação encontrada neste trabalho foi menor que a obtida por Almeida et al. (2009), para o feijão adzuki, cujo valor foi de $31,16 \mathrm{~kJ} \mathrm{~mol}^{-1}$ e maior por o valor relatado por Goneli (2008) para mamona de $15,25 \mathrm{~kJ} \mathrm{~mol}^{-1}$.

\section{Conclusões}

1.Entre os doze modelos analisados, o que apresenta o melhor ajuste para descrever as curvas de secagem do nabo forrageiro é o de Midilli;

2. O coeficiente de difusão efetivo aumenta com a elevação da temperatura do ar de secagem sendo descrito pela equação de Arrhenius e obteve a energia de ativação de $24,78 \mathrm{~kJ} \mathrm{~mol}^{-1}$.

\section{Agradecimentos}

Ao Conselho Nacional de Desenvolvimento Científico e Tecnológico (CNPq) e à Coordenação de Aperfeiçoamento de Pessoal de Nível Superior (CAPES) pelo apoio financeiro indispensável à execução do presente trabalho.

\section{Referências}

AFONSO JÚNIOR, P. C.; CORRÊA, P. C. Comparação de modelos matemáticos para descrição da cinética de secagem em camada fina de sementes de feijão. Revista Brasileira de Engenharia Agrícola e Ambiental, v. 03, n. 03, p. 349-353, 1999.

ALMEIDA, D. P. et al. Cinética de secagem do feijão adzuki (Vigna angularis). Global Science and Technology, v. 02, n. 01, p. 72-83, 2009. 
ANDRADE, E. T. et al. Cinética de secagem e qualidade de sementes de feijão. Engevista, v. 08, n. 02, p. 83-95, 2006.

BABALIS, S. J.; BELESSIOTIS, V. G. Influence of the drying conditions on the drying constants and moisture diffusivity during the thin-layer drying of figs. Journal of Food Engineering, v. 65, n. 03, p. 449-58, 2004.

BASUNIA, M. A., ABE, T. Moisture desorption isotherms of medium-grain rough rice. Journal of Stored Products Research, v. 37, n. 03, p. 205-219, 2001

BROOKER, D. B.; BAKKER-ARKEMA, F. W.; HALL, C. W. Drying and storage of grains and oilseeds. Westport: The AVI Publishing Company, 1992. $450 \mathrm{p}$.

CORRÊA, P. C.; ARAÚJO, E. F.; AFONSO JÚNIOR, P. C. Determinação dos parâmetros de secagem em camada delgada de sementes de milho doce (Zea mays L.). Revista Brasileira de Milho e Sorgo, v. 02, n. 02, p. 110-119, 2003.

CORRÊA, P. C. et al. Modelagem da difusão líquida na secagem do arroz em casca. In:BARBOSA, O. A. Avances en ingeneria agrícola: 2003 - 2005. Villa De Merlo: CADIR, 2005.p. 323-328.

CORRÊA, P. C. et al. Determinação e modelagem das propriedades físicas e da contração volumétrica do trigo, durante a secagem. Revista Brasileira de Engenharia Agrícola e Ambiental, v. 10, n. 03, p. 665-670, 2006.

CORRÊA, P. C. et al. Modelagem matemática para a descrição do processo de secagem do feijão (Phaseolus vulgaris L.) em camadas delgadas. Engenharia Agrícola, v. 27, n. 02, p. 501-510, 2007.

CRUSCIOL, C. A. C. et al. Persistência de palhada e liberação de nutrientes do nabo-forrageiro no plantio direto. Pesquisa Agropecuária Brasileira, v. 40, n. 02, p. 161-168, 2005.

DOMINGOS, A. K. Otimização da etanólise de óleo de Raphanus sativus $L$. e avaliação de sua estabilidade à oxidação. 2005. 113 f. Dissertação (Mestrado em Química) Universidade Federal do Paraná, Curitiba, 2005.

FERRARI, R. A.; OLIVEIRA, V. S.; SCABIO, A. Biodiesel de soja: taxa de conversão em ésteres etílicos, caracterização físico-químicas e consumo em geradores de energia. Química Nova, v. 18, n. 01, p. 19-23, jan. 2005.

FREGOLENTE, L. V. et al. Avaliação das propriedades térmicas efetivas da soja em um secador de leito fixo. Acta Scientiarum, v. 25, n. 01, p. 1-7, 2003.

GONELI, A. L. D. et al. Estudo da difusão de umidade em grãos de trigo durante a secagem. Ciência e Tecnologia de Alimentos, v. 27, n. 01, p. 135-140, 2007

GONELI, A. L. D. Variação das propriedades físicomecânicas e da qualidade de mamona (Ricinus communis L.) durante a secagem e o armazenamento. 2008. $186 \mathrm{f}$. Tese (Doutorado em Engenharia Agrícola). - Universidade Federal de Viçosa, Viçosa, 2008.

GONELI, A. L. D. et al. Cinética de secagem de grãos de café descascado em camada delgada. Revista Brasileira de Armazenamento, v. Especial Café, n. 11, p. 64-73, 2009.
HENDERSON, S. M. Progress in developing the thin layer drying equation. Transactions of the ASAE, v. 17, p. 11671168, 1974.

HENDERSON, S. M., PABIS, S. Grain drying theory: temperature effect on drying coefficient. Journal of Agricultural Engineering Research, v. 06, p. 169-174, 1961.

KARATHANOS, V. T. Determination of water content of dried fruits by drying kinetics Journal of Food Engineering, v. 39, n. 04, p. 337-44, 1999.

KASSEM, A. S. Comparative studies on thin layer drying models for wheat. In: INTERNATIONAL CONGRESS ON AGRICULTURAL ENGINEERING, 13th., 1998, Morocco. [Holdings]. Morocco: [s. n.], 1998. v. 06.

KRZYZANOWSKI, F. C.; WEST, S. H.; FRANÇA NETO, J. B. Drying soybean seed using air ambient temperature at low relative humidity. Revista Brasileira de Sementes, v. 28, n. 02, p. $77-83,2006$

LEWIS, W. K. The drying of solid materials. The Journal of Industrial and Engineering Chemistry, v. 13, n. 05, p. 427-433, 1921.

MADAMBA, P. S.; DRISCOLL, R. H.; BUCKLE, K. A. The Thin-layer drying characteristics of garlic slices. Journal of Food Engineering, v. 29, n. 01, p. 75-97, 1996.

MARTINAZZO, A. P. et al. Análise e descrição matemática da cinética de secagem de folhas de capim-limão. Revista Brasileira de Engenharia Agrícola e Ambiental, v. 11, n. 03, p. 301-306, 2007.

MIDILLI, A.; KUCUK, H.; YAPAR, Z. A New model for single layer drying. Drying Technology, v. 20, n. 07, p. 1503-1513, 2002.

MOHAPATRA, D.; RAO, P. S. A Thin layer drying model of parboiled wheat. Journal of Food Engineering, v. 66, n. 04, p. 513-518, 2005.

MOHSENIN, N. N. Physical properties of plant and animal materials. New York: Gordon and Breach Publishers, 1986.

PAGE, G. E. Factors influencing the maximum rates of air drying shelled corn in thin layers. West Lafayette: Purdue University, 1949.

PANCHARIYA, P. C.; POPOVIC, D.; SHARMA, A. L. Thinlayer modeling of black tea drying process. Journal of Food Engineering, v. 52, n. 04, p. 349-357, 2002.

QUEIROZ, D. M.; PEREIRA, J. A. M.; MELO, E. C. Modelos matemáticos de simulação de secagem de grãos. Viçosa: Centro Nacional de Treinamento em Armazenagem, 1995. 59 p.

RAMESH, M. N. Moisture transfer properties of cooked rice during drying. Lebensmittel-Wissenschaft und-Technologie, v. 36, n. 02, p. 245-255, 2003.

RESENDE, O. et al. Contração volumétrica na difusão líquida durante o processo de secagem do arroz em casca. Revista Brasileira de Armazenamento, v. 30, n. 02, p. 63-171, 2005.

RESENDE, O. et al. Modelagem matemática do processo de secagem de duas variedades de feijão (Phaseolus vulgaris L.). 
Revista Brasileira de Produtos Agroindustriais, v. 10, n. 01, p. 17-26, 2008.

RESENDE, O. et al. Modelagem matemática para a secagem de clones de café (Coffea canephora Pierre) em terreiro de concreto. Acta Scientiarum. Agronomy, v. 31, n. 02, p. 189-196, 2009.

SANTOS, C. J. R. Secagem de sementes de girassol via radiação infravermelho e convecção forçada de ar aquecido. 2009. 75 f. Dissertação (Mestrado em Engenharia de Processos). - Universidade Tiradentes, Aracaju, 2009.

SHARAF-ELDEEN, Y. I.; BLAISDELL, J. L.; HAMDY, M. Y. A model for ear corn drying. Transactions of the ASAE, v. 23, p. 1261-1265, 1980.

SHARMA, G. P; PRASAD, S. Effective moisture diffusivity of garlic cloves undergoing microwave-convective drying, Journal of Food Engineering, v. 65, n. 04, p. 609-617, 2004.

THOMPSON, T. L.; PEART, R. M.; FOSTER, G. H. Mathematical simulation of corn drying: a new model. Transactions of ASAE, v. 11, n. 04, p. 582-586, 1968.
ULLMANN, R. et al. Qualidade das sementes de pinhão manso submetidas à secagem artificial. Revista Ciência Agronômica, v. 41, n. 03, p. 442-447, 2010.

VERMA, L. R. et al. Effects of drying air parameters on rice drying models. Transactions of the ASAE, v. 28, p. 296301, 1985.

YALDIZ, O.; ERTEKIN, C.; UZUN, H. I. Mathematical modeling of thin layer solar drying of sultana grapes. Energy, v. 26, p. 457-465, 2001.

YAGCIOGLU, A.; DEGIRMENCIOGLU, A.; CAGATAY, F. Drying characteristics of laurel leaves under different conditions. In: INTERNATIONAL CONGRESS ON AGRICULTURAL MECHANIZATION AND ENERGY, 7th., 1999, Adana. Proceedings... Adana: Cukurova University, 1999. p. 565-569.

WANG, C. Y.; SINGH, R. P. Use of variable equilibrium moisture content in modeling rice drying. Transaction of ASAE, v. 11, p. 668-672. 